\title{
Effects of treatment with androgen receptor ligands on microRNA expression of prostate cancer cells
}

Corrinne V Segal ${ }^{1}$, Costas Koufaris $^{2}$, Chris Powell $^{3}$, Nigel J Gooderham ${ }^{1 *}$

${ }^{1}$ Department of Surgery \& Cancer, Biomolecular Medicine, Imperial College London, London SW72AZ, UK

${ }^{2}$ Department of Cytogenetics and Genomics, Cyprus Institute of Neurology and

Genetics, Cyprus

${ }^{3}$ GlaxoSmithKline, Ware, United Kingdom

*Correspondence to:

Nigel J Gooderham

Computational and Systems Medicine,

Surgery and Cancer,

Sir Alexander Fleming Building

Imperial College London,

London,

UK, SW7 2AZ

Tel: (+44) 02075943188

Email: n.gooderham@imperial.ac.uk

Keywords: androgen agonist, androgen antagonist, prostate cancer, miR-210, miR221

Abbreviations: microRNA (miRNA), androgen receptor (AR), bicalutamide (BIC), dihydrotestosterone (DHT), mibolerone (MIB), prostate-specific antigen (PSA), androgen responsive elements (AREs), vascular endothelial growth factor (VEGF), epithelial growth factor (EGF) 


\begin{abstract}
Post-transcriptional regulation by microRNA (miRNA) is an important aspect of androgen receptor (AR) signalling in prostate cancer cells. However, the global profiling of miRNA expression in prostate cancer cells following treatment with AR ligands has not been reported so far. In this study we examined the effect of treatment with two AR agonists (mibolerone (MIB) and dihydrotestosterone (DHT)) and an AR antagonist (bicalutamide (BIC)) on miRNA expression in the human androgendependent $\mathrm{LNCaP}$ prostate cancer cell line using microarray technology and verification of selected miRNA using quantitative real-time PCR (qRT-PCR). No miRNA were identified as differentially expressed following treatment with the AR antagonist BIC. In contrast, a number of common and compound-specific alterations in miRNA expression were observed following treatment with AR agonists. Unexpectedly it was found that treatment with the AR agonists resulted in the repression of miR-221, a miRNA previously established to be involved with prostate cancer development. This observation indicates that this miRNA may have a more complex role in prostate cancer development than considered previously. Treatment with MIB led to an induction of miR-210 expression, a hypoxia-related miRNA. This miRNA is reported to be involved in cell adaptation to hypoxia and thus induction in conditions of normoxia may be important in driving metabolic changes observed in prostate cancer. Thus examining the effect of AR agonists and antagonists on miRNA expression can provide novel insights into the response of cells to AR ligands and subsequent downstream events.
\end{abstract}




\section{Introduction}

The androgen receptor (AR) is a member of the steroid hormone receptor family that is expressed in various types of tissues. Activation of AR intracellular signalling pathways by natural androgens such as testosterone and dihydroxytestosterone (DHT) is necessary for the development and maintenance of male characteristics in vertebrates (Heinlein \& Chang, 2002). The prostate in particular is an organ that requires the action of androgens on the AR in order to develop and function. The dependency of prostate cells on androgen-mediated AR signalling for development and maintenance is also retained in the majority of prostate tumours (Heinlein \& Chang, 2004). AR antagonists inhibit the effect of AR, acting by a variety of mechanisms such as competitive binding to the AR and the blocking of androgen synthesis. Given the dependency of prostate cancer cells on AR signalling for growth and survival, AR inhibitors are valuable clinical tools for the treatment of prostate cancer patients (Heinlein \& Chang, 2004).

It is being increasingly recognized that post-transcriptional regulation by non-coding miRNA is an important mechanism by which the activated AR modulates the phenotype of prostate cells. In the setting of prostate cancer AR regulated miRNA have been reported to be associated with the development and severity of the disease. Examples include the miR-99/let-7c/miR-125b miRNA cluster involved in the regulation of multiple androgen-induced genes (Sun et al., 2014), the epigenetically repressed miR-31 which is a direct regulator of the AR (Lin et al., 2013), and miR221 which has been linked to the progression of prostate cancer cells to a state of androgen independence (Sun et al. 2009; Sun et al., 2014). Studies aiming to investigate the role of miRNA in AR signalling and prostate cancer have taken a variety of approaches. These include the identification of miRNA that target the AR receptor (Östling et al., 2011), comparison of androgen sensitive and insensitive cell lines (Ottman et al., 2014), comparison of miRNA expression between metastatic and non-metastatic prostate cancer cell lines (Saini et al., 2011), and examination of miRNA expression in prostate tumour samples (Szczyrba et al., 2010). 
In this study we used a different approach, investigating the effect of direct treatment cells, an androgen-dependent prostate cancer cell line, using miRNA microarray followed by verification of individual differentially expressed miRNAs using qRTPCR. This approach has a number of advantages. Firstly, it allows the direct comparison of the effect of treatment with $\mathrm{AR}$ agonists and antagonists on the miRNA expression of prostate cancer cell lines. Secondly, it can be used to determine compound-specific effects on miRNA expression. Lastly, it allows the identification of novel AR-regulated miRNA and miRNA implicated in the AR regulatory network. We have used DHT, the most potent endogenous androgenic compound (Luu-The V et al., 2008) and mibolerone (MIB), a potent non-metabolisable synthetic androgen agonist, originally used by veterinarians to prevent oestrus in female dogs (Sokolowski and Geng, 1977). MIB has been used extensively in cell culture assays to study effects of the AR. Additionally we have examined bicalutamide (BIC), also known as Casodex ${ }^{\circledR}$, a therapeutic non-steroidal androgen antagonist used as part of a regimen in the treatment of androgen-sensitive prostate cancer (Fradet, 2004).

\section{Materials \& Methods}

\section{Cell culture}

All cell culture reagents were supplied by Life Technologies (Paisley, UK) unless otherwise stated, and cells were propagated in Corning® standard tissue culture plastic-ware. Human LNCaP cells were purchased from ATCC at passage number 17 and experiments were conducted within 10 passages of the original number. LNCaP were routinely cultured in complete media consisting of phenol red-free minimum essential medium supplemented with $10 \% \mathrm{v} / \mathrm{v}$ foetal bovine serum (FBS), $1 \% \mathrm{v} / \mathrm{v}$ non-essential amino acids, $2 \mathrm{mM}$ L-glutamine, and $1 \%$ Penicillin/Streptomycin (100U/mL, $100 \mu \mathrm{g} / \mathrm{mL}$ respectively).

\section{Cell treatments}


BIC and DHT were purchased from Sigma-Aldrich (Gillingham, UK). MIB was obtained from Perkin Elmer (Waltham, MA, USA). Prior to administration of the treatments LNCaP cells were plated at 50,000 cells $/ \mathrm{mL}$ in steroid-depleted (stripped) media for 2 days, to reduce the amount of endogenous steroids and growth factors, thus potentially lowering the total testosterone by as much as $80 \%$ (Sedelaar and Isaacs, 2009). The actions of exogenous steroids can then be assessed without influence from endogenous steroids. The dextran-coated charcoal stripped FBS (dccFBS) was generated as described previously (Papaioannou et al., 2014) and added to the experimental media at a concentration of $5 \%$.

\section{Cell Proliferation}

Cell numbers were assessed using a haemocytometer and viability assessed using trypan blue exclusion or using AlamarBlue ${ }^{\circledR}$ assay (AbD Serotec, 2009). AlamarBlue ${ }^{\circledR}$ indicator dye changes colour and fluoresces in response to the chemical reduction of resazurin to fluorescent resorufin by metabolically competent cells (AbD Serotec, 2009); the amount of reduction is directly proportional to the number of cells present in the sample. Samples were examined at various times after treatment.

\section{Enzyme-linked immunosorbent assay (ELISA) assays}

PSA expression was measured using PSA enzyme-linked immunosorbent assay (ELISA) kit PS067T as per the manufacturer's protocol (Calbiotech, Spring Valley, CA, USA). The amount of PSA was determined by comparison to the standard curve which was run concurrently with each set of samples. The cellular supernatant containing the secreted PSA, diluted with distilled water as required, was used to assess PSA secretion. Samples were examined at various times after treatment.

EGF expression was measured using Human EGF ELISA kit KHG0061 as per the manufacturer's recommendations (Life Technologies). EGF expression was determined by comparison to a standard curve. 


\section{Hypoxia culturing conditions}

Based on previous reports (Giannakakis et al., 2008) 16 hours of $1 \% \mathrm{O}_{2}$ was used to induce hypoxia in LNCaP. Cells were grown to approximately $70 \%$ confluence, the media was refreshed (normal culturing media) and flasks transferred to $1 \% \mathrm{O}_{2}$ (hypoxia) or maintained in control conditions $\left(21 \% \mathrm{O}_{2}\right.$ normoxia). After 16 hours, the normoxic and hypoxic cultures were harvested for RNA and protein extraction.

\section{RNA extraction}

To prevent RNA degradation by RNAses, all reagents were certified RNase-free and RNaseZap ${ }^{\circledR}$ (Life Technologies) was used to decontaminate surfaces and pipettes. Isolation of RNA from cells was performed using TRIzol® (Life Technologies) following the manufacturer's instructions. RNA pellets were re-suspended in $40 \mu \mathrm{L}$ of Tris/EDTA (TE) buffer pH 8.0 and assessed for concentration and quality. All RNA samples used for subsequent work had 260/280 ratios greater than 2.0 and 260/230 ratios greater than 1.8. Additionally, an RNA integrity number (RIN) was generated for each sample using the RNA Nano reagents and an Agilent Bioanalyser (Agilent Technologies, Santa Clara, CA, USA). All samples used for subsequent work had RIN greater than 7.6.

\section{miRNA microarrays}

Profiling of global miRNA expression in $\mathrm{LNCaP}$ was performed using the Agilent Human miRNA Microarray kit (V3) and microarray platform as described previously (Koufaris et al., 2012). On each microarray 851 human miRNAs were present, consisting of 680 dominant miRNA and $171 *$ miRNA.

\section{Microarray pre-processing and normalisation}

Processing and normalisation of the microarray data were conducted in the $\mathrm{R}$ statistical programming language ( $\mathrm{R}$ version 13.2; http://www.r-project.org), using the "AgiMicroRna" software package, version 2.2, available as part of the BioConductor 
Project (http://www.bioconductor.org). miRNAs were retained in the analysis if they condition (treatment and time) and above the threshold (negative control expression level plus $1.5 \mathrm{x}$ standard deviation) in at least $25 \%$ of samples within at least one experimental condition. The microarray data were normalised using the robust multiarray average method with quantile normalisation. ComBat was used to reduce batch effects (Johnson et al., 2007).

\section{Microarray analysis}

For data collected over multiple time points, time course analysis was conducted in AgiMicroRna using Limma with the method of Benjamini-Hochberg used to control the false discovery rate to $30 \%$

\section{Quantitative reverse transcription polymerase chain reaction (qRT-PCR)}

qRT-PCR was used to verify differentially expressed miRNAs identified by microarray, For mature miRNA qRT-PCR reactions, pre-designed Taqman probes were used (Applied Biosystems). The PCR reactions were performed in an ABI Prism 7700 Sequence Detection System. All PCR reactions were run in triplicate to provide technical replicates. Relative quantification was performed using the $2^{-\Delta \Delta \mathrm{CT}}$ method and using RNU6B as the reference gene. In addition to the no-RT, a 'no-template control' (no-TC) was also run to ensure the reagents were free of contaminants.

The Applied Biosystems High Capacity cDNA reverse transcription kit with RNase inhibitor (Part number 4374966) with the VEGF TaqMan® Gene Expression Assay (Hs00900055_m1) or TaqMan Ribosomal RNA Control Reagents was employed to measure VEGF and 18S levels, respectively.

\section{Results}


Effects of DHT, MIB, and BIC treatments on proliferation and PSA secretion in LNCaP cells

Two established end-points and surrogate markers of AR activation in prostate cells are increased proliferation and PSA secretion. We initially examined the effects of DHT, MIB, and BIC on proliferation (Suppl.Fig.1A-C) and PSA secretion (Suppl.Fig.2A-C) in LNCaP cells. In addition, the ability of a range of BIC doses to inhibit DHT-induced proliferation (Suppl.Fig.1D) and PSA secretion (Suppl.Fig.2D) were also examined. As expected DHT and MIB treatments induced dose-dependent increases in LNCaP proliferation and PSA secretion. Whilst BIC treatment alone induced a madest increase in proliferation and PSA secretion, in the presence of DHT it had its reparted antagunistic effect. The madest induction by BIC may be due to a mismatch between the availability of co-regulators and the amount required far full antaganism (Hadgsan et al, 2007).

Based on these results a single concentration of each compound was selected for subsequent study (BIC $10 \mu \mathrm{M}$ - therapeutically relevant concentration, DHT $2 \mathrm{nM}-$ physiologically relevant concentration, MIB 100pM - induction of maximal proliferative response). The effects of these doses were examined at multiple time points after treatment initiation (4, 8, 24 and 120 hours) in order to determine temporal changes in cell number, PSA secretion, and miRNA expression. The number of LNCaP cells at each treatment time point was first determined (Fig.1A). BIC treatment resulted in a similar trend as the vehicle control, namely a slight increase in cell number over the study period, although at 120 hours of BIC treatment there was a statistically significant decrease in cell number (18\% reduction compared to vehicle control; $\mathrm{p}<0.05)$. In the case of DHT a time-dependent increase in cell number was observed, reaching a statistically significant increase above the time-matched control at 120 hours $(122 \%$ increase compared to vehicle control; $\mathrm{p}<0.01)$. Treatment with MIB elicited an even more prominent increase in cell number, reaching statistical significance at 120 hours $(190 \%$ compared to vehicle, $\mathrm{p}<0.001)$.

In conjunction with the assessment of cell numbers, PSA secretion was assayed at each time point (Fig.1B). In BIC and vehicle treated cells a trend for a steady increase in PSA over time was observed. As expected the two AR agonists caused prominent increases in PSA secretion. There was a 7.3-fold increase and 10.3-fold increase in 
DHT-treated cells compared with the control at 24 hours and 120 hours, respectively. hours, PSA was 36.2 times higher than observed in the time-matched control.

\section{Microarray profiling of miRNA expression}

Having characterised the phenotypic response of $\mathrm{LNCaP}$ cells to the AR ligands (Fig.1; Suppl.Fig.1-2), we examined the potential role of miRNA in these responses. Microarray analysis was performed on 3 independent cultures per data point to determine the temporal nature of global miRNA expression profiles in the treated cells. Differentially expressed miRNA (DEM) were identified by performing a time course analysis, implemented in the AgiMicroRna package. This methodology allowed the identification of miRNAs which respond differently over time in each treatment group relative to the control. Treatment with the AR antagonist BIC did not significantly alter the expression of miRNA at any timepoint compared with the timematched control. In contrast, treatment with the AR agonists MIB and DHT resulted in significant changes in the expression of miRNA (Tables 1-2). All the miRNA affected by DHT were found to respond at 24 hours of treatment, with their deregulation being consistent at 120 hours. For MIB, miRNA were only determined to be affected by the treatment at 120 hours. Some of the identified DEM were affected by both AR agonists (miR-221, miR-99a, miR-1246, and miR-1290), while others were affected uniquely by one of the tested compounds (e.g. miR-210 for MIB and miR-494 for DHT).

To validate the reliability of the microarray data qRT-PCR was used to assess the expression levels of five miRNA in the same samples used for microarray analysis. This analysis found a high degree of concordance between the levels of miRNA determined by qRT-PCR and microarrays, verifying the reliability of the microarray data (Fig.2).

\section{Expression of miR-221}

A defining feature of MIB treatment of LNCaP cells was the significant decrease in the expression of miR-221, also seen in DHT-treated cells, but less pronounced 
(Tables 1 and 2). Increased expression of miR-221 has been shown to be involved in prostate cancer development and the progression to AR agonist independence (Gilardi et al., 2007; Sun et al., 2009; Kneitz et al., 2014; Sun et al., 2014). Our findings showing a decrease in expression are consistent with the AR responsiveness of LNCaP cells. Thus understanding this responsiveness may offer insights into the role of miR-221 in the progression of AR agonist independence. To investigate this interaction further we examined how expression of miR-221 relates to exposure to a range of MIB doses (from 1pM to 10nM). As expected MIB treatment resulted in a concentration dependent induction of LNCaP proliferation and PSA secretion (Fig.3A). Conversely, MIB treatment resulted in a concentration-dependent decrease in the expression of miR-221, up to 4-fold (Fig.3B). In these conditions, both PSA and proliferation were significantly inversely correlated with miR-221 expression ( $\mathrm{r}=-$ 0.8780 and -0.9053 , respectively, $\mathrm{p}<0.05)$.

\section{Expression of miR-210}

Among the miRNA displaying significant expression changes in response to MIB treatments was miR-210, a miRNA known to be upregulated by hypoxia and involved in driving cellular adaptations to low oxygen levels (Camps et al., 2008; Chan et al., 2009). Indeed, we verified that in the absence of MIB, miR-210 levels in LNCaP cells were increased following hypoxia (Fig.4A). As a positive control it was also demonstrated that this treatment also caused the induction of the prototypical hypoxia responsive gene, the vascular endothelial growth factor (VEGF) (Fig.4B).

To examine further the link between AR activation and miR-210 induction we examined the effect of doses of MIB on expression of this miRNA (Fig.4C) using qRT-PCR. Induction of miR-210 was observed in response to high doses of MIB $(1 \mathrm{nM}$ and $10 \mathrm{nM})$, but not as a result to treatment with lower doses. MIB treatment also resulted in increased levels of VEGF (Fig.4D), with miR-210 and VEGF displaying significantly correlated increases following MIB treatment (Pearson correlation 0.86, $\mathrm{P}<0.05$, Fig.4E).

Mabjeesh et al., 2003 reported that treatment of LNCaP cells with AR agonists was able to induce the HIF1 $\alpha$ protein in conditions of normoxia via an autocrine loop involving increased epidermal growth factor (EGF) production and secretion. This 
was therefore considered a potential mechanism by which MIB could induce miR-210 and VEGF in conditions of normoxia. However, no EGF was detected in the cell media in LNCaP cells treated with vehicle or various MIB doses (Suppl.Fig.3).

\section{Discussion}

In this study we examined the effect of treatment with $\mathrm{AR}$ agonists and an $\mathrm{AR}$ antagonist on miRNA expression in the androgen-dependent $\mathrm{LNCaP}$ prostate cancer cell line. To the best of our knowledge this is the first study to directly investigate the temporal effects of AR agonists and antagonists on global miRNA profiles in prostate cancer cells. One obvious limitation of this approach is that it does not take into account the role of non-parenchymal cells in modulating the prostate response to AR signalling. Nevertheless, although in vitro studies can only replicate limited aspects of the in vivo response to androgens, valuable insights can still be obtained in regards to the involvement of miRNA to AR signalling and thus potentially to prostate cancer development. Another limitation of this study is that for microarray miRNA profiling a single concentration of each compound was studied, at different phases of the proliferation curve, and therefore in future studies it would be of use to study these compounds at concentrations of similar potency.

An intuitive effect of AR antagonists on miRNA expression in prostate cancer cells would be the reversal of the effects of AR agonists. However, it is also possible that AR antagonists have additional effects on miRNA due to the recruitment of alternative co-regulators, activators and repressors, to the AR or by acting through AR independent pathways. An interesting hypothesis is that miRNA specifically affected by $\mathrm{AR}$ antagonists can have important phenotypic consequences, such as being involved in the progression of prostate cancer cells to a state of AR independence. To identify potential subsets of miRNA specifically affected by AR antagonists we examined LNCaP cells, cultured in the absence of AR agonists, treated with BIC. The BIC treatment used here was demonstrated to be able to inhibit AR-agonist induced proliferation of LNCaP cells (Suppl.Fig.1-2). Nevertheless, in this study no miRNA were identified as being significantly differentially expressed due to BIC treatment alone. These findings are consistent with the phenotypic effects of BIC being the antagonist of AR agonists, with few, if any, distinct effects on miRNA. However, 
further investigations with more compounds and in different cell lines are required to expression that go beyond the reversal of AR agonism.

In contrast to BIC, the AR agonists DHT and MIB had clear effects on the expression of miRNA in the LNCaP cells (Tables 1-2). Some of the identified miRNA were differentially expressed in response to treatment with both DHT and MIB, while others were responsive to only one of the tested compounds. It is quite likely that the majority of miRNA affected by both AR agonists are under direct regulation by the $\mathrm{AR}$ or are associated with the phenotypic changes induced by the compounds. One of the commonly affected miRNA identified here was miR-99a. This miRNA has been demonstrated previously to be under direct transcriptional regulation of the AR and to be involved in the direct post-transcriptional regulation of a number of AR responsive genes (Sun et al., 2014). This miRNA is also a suppressor of proliferation and PSA in prostate cells (Sun et al., 2011). The repression of this miRNA observed following treatment with both MIB and DHT could contribute to the observed increase in proliferation and PSA secretion of LNCaP cells (Fig.1).

An interesting finding was that both DHT and MIB treatment caused a significant downregulation of miR-221, a miRNA strongly implicated in prostate cancer (Tables 1-2; Fig.3). Higher expression of miR-221 has been linked to the progression of prostate cancer cells from androgen-dependency to androgen-independent growth (Sun et al., 2009; Sun et al., 2014), with greater proliferation and aggressiveness of prostate cancer cell lines (Galardi et al., 2007), and with worse prognosis in high-risk prostate cancer patients (Kneitz et al., 2014). However, previous studies had not determined how expression of this miRNA is affected by exposure to AR agonists. Unexpectedly, in this study we have found that treatment with AR agonists caused the reduced expression of oncogenic miR-221 in the LNCaP cell line. Interestingly, the sensitivity of miR-221 to MIB doses appears to be the reverse of the effects of the chemical on cell numbers and PSA secretion (Fig.3). This observation indicates a more complex role for miR-221 in prostate cancer development than previously recognised. One possible interpretation of this observation is that $\mathrm{AR}$ signalling mediated the repression of miR-221 as a mechanism of maintaining proliferative prostate cells in a state of androgen-dependency. In this case these cells will then 
return to a quiescent state more rapidly when the AR signalling is terminated. Further experimental work is required to determine the relevance and importance of the ARmiR-221 interaction in vivo.

Another interesting miRNA that was affected by both DHT and MIB was miR-1290. This miRNA has previously been reported to be a circulating prognostic marker for castration-resistant prostate cancer (Huang et al., 2014). This miRNA has been linked to inhibition of the cell cycle and promotion of apoptosis in neuronal and lung carcinoma cells by targeting p27 and BCL2 (Yelamanchili et al., 2014; Kim et al.2014). Interestingly, this is an evolutionary young, great-ape specific miRNA (Yelamanchili et al., 2014). Such species-specific miRNA repertoire may be important determinants of differential species responses to equivalent xenobiotic exposures such as AR agonists (Koufaris \& Gooderham, 2013).

We also determined in this study miRNA responses that were specific to only one of the tested compounds. Such compound-specific effects on miRNA could be due to a variety of technical reasons e.g. different potency of AR activation. It is also known that AR agonists can also have distinct biological effects. For example, in addition to effects on AR, MIB can also activate the progesterone receptor besides the AR, which is not the case for DHT (Cops et al., 2008). The differential effects of AR agonists on miRNA could potentially be important in determining differential effects of compounds on prostate cells or assist in identifying mode of action of such chemicals.

An interesting example of a compound specific miRNA response identified here was the induction of miR-210 following MIB treatment (Fig.4). This miRNA is activated by hypoxia and is involved in driving cell adaptations to lack of oxygen (Giannakakis et al., 2008; Chan et al., 2009). In a previous study it has been reported that expression of miR-210 is induced in prostate cancer cell lines exposed to hypoxia (Quero et al., 2010). However, here it was demonstrated that MIB could induce this miRNA in cell culture conditions with adequate oxygen concentrations, suggesting functions for this miRNA beyond its established role in hypoxia. The effect on miR210 was only observed at high doses of MIB (Fig.4). Consequently the lack of response to DHT in this case may reflect the lower potency of the compound to activate the AR. We did not clarify here the mechanism by which AR agonists can 
activate miR-210 in conditions of normoxia, but activation of miR-210 in vivo could be important in driving the metabolic changes observed in prostate cancer (Vaughan et al., 2013).

Prostate cancer is currently the most commonly diagnosed cancer in males and a prevalent cause of global mortality. Consequently there is considerable interest in delineating the molecular mechanisms implicated in the initiation and progression of the disease. Research into the contribution of miRNA to prostate cancer development holds considerable promise. Firstly, miRNA that are key mediators of AR signalling could be novel therapeutic targets (Deng et al., 2013). Secondly, circulating miRNA could prove to be easily accessible biomarkers for the disease, either in plasma (Lin et al., 2014) or in prostate fluid (Selth et al., 2014). This study demonstrates that direct profiling of the effects of AR agonists and antagonists on miRNA expression is a methodological approach that can assist towards clarifying the role of these genes in AR signalling and prostate cancer. 


\section{References}

AbD Serotec (2009). BUF012A/B alamarBlue® Product Datasheet. Available from http://static.abdserotec.com/uploads/BUF012-technical-datasheet-2011.pdf [Accessed 01.07.2012]

Camps C, Buffa FM, Colella S, Moore J, Sotiriou C, Sheldon H, Harris AL, Gleadle JM, Ragoussis J. hsa-miR-210 is induced by hypoxia and is an independent prognostic factor in breast cancer. Clin Cancer Res. 2008 1;14(5):1340-8.

Chan SY, Zhang YY, Hemann C, Mahoney CE, Zweier JL, Loscalzo J. MicroRNA210 controls mitochondrial metabolism during hypoxia by repressing the iron-sulfur cluster assembly proteins ISCU1/2. Cell Metab. 2009 10(4):273-84.

Cops EJ, Bianco-Miotto T, Moore NL, Clarke CL, Birrell SN, Butler LM, Tilley WD. Antiproliferative actions of the synthetic androgen, mibolerone, in breast cancer cells are mediated by both androgen and progesterone receptors. J Steroid Biochem Mol Biol. 2008 ;110(3-5):236-43.

Deng JH, Deng Q, Kuo CH, Delaney SW, Ying SY. MiRNA targets of prostate cancer. Methods Mol Biol. 2013;936:357-69.

Fradet Y. Bicalutamide (Casodex) in the treatment of prostate cancer. Expert Rev Anticancer Ther. 2004 Feb;4(1):37-48.

Galardi S, Mercatelli N, Giorda E, Massalini S, Frajese GV, Ciafrè SA, Farace MG. miR-221 and miR-222 expression affects the proliferation potential of human prostate carcinoma cell lines by targeting p27Kip1. J Biol Chem. 2007 10;282(32):23716-24.

Giannakakis A, Sandaltzopoulos R, Greshock J, Liang S, Huang J, Hasegawa K, Li C, O'Brien-Jenkins A, Katsaros D, Weber BL, Simon C, Coukos G, Zhang L. miR210 links hypoxia with cell cycle regulation and is deleted in human epithelial ovarian cancer. Cancer Biol Ther. 2008 7(2): 255-264. 
Heinlein CA, Chang C. Androgen receptor (AR) coregulators: an overview. Endocr Rev. 2002 23(2):175-200.

Heinlein CA, Chang C. Androgen receptor in prostate cancer. Endocr Rev. 2004 25(2):276-308.

Hodgson MC, Astapova I, Hollenberg AN, Balk SP (2007). Activity of androgen receptor antagonist bicalutamide in prostate cancer cells is independent of NCoR and SMRT corepressors. Cancer Res 67(17): 8388-8395.

Huang X, Yuan T, Liang M, Du M, Xia S, Dittmar R, Wang D, See W, Costello BA, Quevedo F, Tan W, Nandy D, Bevan GH, Longenbach S, Sun Z, Lu Y, Wang T, Thibodeau SN, Boardman L, Kohli M, Wang L. Exosomal miR-1290 and miR-375 as Prognostic Markers in Castration-resistant Prostate Cancer. Eur Urol. 2014 13. pii: S0302-2838(14)00687-3.

Johnson WE, Li C, Rabinovic A (2007). Adjusting batch effects in microarray expression data using empirical Bayes methods. Biostatistics 8(1): 118-127.

Kim KB, Kim K, Bae S, Choi Y, Cha HJ, Kim SY, Lee JH, Jeon SH, Jung HJ, Ahn KJ, An IS, An S. MicroRNA-1290 promotes asiatic acid-induced apoptosis by decreasing BCL2 protein level in A549 non-small cell lung carcinoma cells. Oncol Rep. 2014 32(3):1029-36.

Kneitz B, Krebs M, Kalogirou C, Schubert M, Joniau S, van Poppel H, Lerut E, Kneitz S, Scholz CJ, Ströbel P, Gessler M, Riedmiller H, Spahn M. Survival in patients with high-risk prostate cancer is predicted by miR-221, which regulates proliferation, apoptosis, and invasion of prostate cancer cells by inhibiting IRF2 and SOCS3. Cancer Res. 2014 1;74(9):2591-603.

Koufaris C, Wright J, Currie RA, Gooderham NJ. Hepatic microRNA profiles offer predictive and mechanistic insights after exposure to genotoxic and epigenetic hepatocarcinogens. Toxicol Sci. 2012 128(2):532-43.

Koufaris C, Gooderham NJ. Are differences in microRNA regulation implicated in species-dependent response to toxicological exposures? Toxicol Sci. 2013 131(2):337-42. 
Lin PC, Chiu YL, Banerjee S, Park K, Mosquera JM, Giannopoulou E, Alves P, MA. Epigenetic repression of miR-31 disrupts androgen receptor homeostasis and contributes to prostate cancer progression. Cancer Res. 2013 1;73(3):1232-44.

Lin HM, Castillo L, Mahon KL, Chiam K, Lee BY, Nguyen Q, Boyer MJ, Stockler MR, Pavlakis N, Marx G, Mallesara G, Gurney H, Clark SJ, Swarbrick A, Daly RJ, Horvath LG. Circulating microRNAs are associated with docetaxel chemotherapy outcome in castration-resistant prostate cancer. Br J Cancer. 2014 13;110(10):246271.

Luu-The V, Bélanger A, Labrie F. Androgen biosynthetic pathways in the human prostate. Best Prac Res Cl En. 2008 22(2): 207-221.

Mabjeesh NJ, Willard MT, Frederickson CE, Zhong H, Simons JW. Androgens stimulate hypoxia-inducible factor 1 activation via autocrine loop of tyrosine kinase receptor/phosphatidylinositol 3'-kinase/protein kinase B in prostate cancer cells. Clin Cancer Res. 2003 9(7):2416-25.

Östling P, Leivonen SK, Aakula A, Kohonen P, Mäkelä R, Hagman Z, Edsjö A, Kangaspeska S, Edgren H, Nicorici D, Bjartell A, Ceder Y, Perälä M, Kallioniemi O. Systematic analysis of microRNAs targeting the androgen receptor in prostate cancer cells. Cancer Res. 2011 1;71(5):1956-67.

Ottman R, Nguyen C, Lorch R, Chakrabarti R. MicroRNA expressions associated with progression of prostate cancer cells to antiandrogen therapy resistance. Mol Cancer. 2014 3;13:1.

Papaioannou MD, Koufaris C, Gooderham NJ. The cooked meat-derived mammary carcinogen 2-amino-1-methyl-6-phenylimidazo[4,5-b]pyridine (PhIP) elicits estrogenic-like microRNA responses in breast cancer cells. Toxicol Lett. 2014 17;229(1):9-16.

Quero L, Dubois L, Lieuwes NG, Hennequin C, Lambin P. miR-210 as a marker of chronic hypoxia, but not a therapeutic target in prostate cancer. Radiother Oncol. 2011 Oct;101(1):203-8. 
Saini S, Majid S, Yamamura S, Tabatabai L, Suh SO, Shahryari V, Chen Y, Deng G,

Sedelaar JP, Isaacs JTC. Tissue culture media supplemented with $10 \%$ fetal calf serum contains a castrate level of testosterone. Prostate 2009 69(16): 1724-1729.

Selth LA, Roberts MJ, Chow CW, Marshall VR, Doi SA, Vincent AD, Butler LM, Lavin MF, Tilley WD, Gardiner RA. Human seminal fluid as a source of prostate cancer-specific microRNA biomarkers. Endocr Relat Cancer 2014 21(4):L17-21.

Sokolowski JH, Geng S. Biological evaluation of mibolerone in the female Beagle. Am J Vet Res. 1977 38(9): 1371-1376.

Sun T, Wang Q, Balk S, Brown M, Lee GS, Kantoff P. The role of microRNA-221 and microRNA-222 in androgen-independent prostate cancer cell lines. Cancer Res. 2009 15;69(8):3356-63.

Sun D, Lee YS, Malhotra A, Kim HK, Matecic M, Evans C, Jensen RV, Moskaluk CA, Dutta A. miR-99 family of MicroRNAs suppresses the expression of prostatespecific antigen and prostate cancer cell proliferation. Cancer Res. 2011 15;71(4):1313-24.

Sun T, Wang X, He HH, Sweeney CJ, Liu SX, Brown M, Balk S, Lee GS, Kantoff PW. MiR-221 promotes the development of androgen independence in prostate cancer cells via downregulation of HECTD2 and RAB1A. Oncogene 2014 May 22;33(21):2790-800.

Szczyrba J, Löprich E, Wach S, Jung V, Unteregger G, Barth S, Grobholz R, Wieland W, Stöhr R, Hartmann A, Wullich B, Grässer F. The microRNA profile of prostate carcinoma obtained by deep sequencing. Mol Cancer Res. 2010 Apr;8(4):529-38.

Vaughan RA, Garcia-Smith R, Trujillo KA, Bisoffi M. Tumor necrosis factor alpha increases aerobic glycolysis and reduces oxidative metabolism inprostate epithelial cells. Prostate 2013;73(14):1538-46. 
Yelamanchili SV, Morsey B, Harrison EB, Rennard DA, Emanuel K, Thapa I, Bastola DR, Fox HS. The evolutionary young miR-1290 favors mitotic exit and differentiation of human neural progenitors through altering the cell cycle proteins. Cell Death Dis. 2014 Jan 9;5:e982. 
Fig.1 Effects of BIC, DHT, and MIB treatments on proliferation and PSA secretion in LNCaP cells over time (A) Effect of vehicle control and DHT, MIB, and BIC on LNCaP cell numbers with time; (B); Effect of vehicle control and DHT, MIB, and BIC on LNCaP PSA secretion with time. Presented as the mean of three or four independent cultures with S.D. error bars. Statistical analysis was performed by 1way ANOVA with Dunnett's post hoc test comparing to the vehicle control time point, where $* * *$ indicates $\mathrm{P}<0.001, * * \mathrm{P}<0.01$ and $* \mathrm{P}<0.05$.

Fig.2 qRT-PCR verification of microarray results for AR agonist treated cells. On the Y-axis the mean hybridization values for the selected miRNA as measured using microarrays are plotted. On the X-axis the mean values for the same miRNA as determined by qRT-PCR are plotted. Pearson's correlation is indicated. Each individual point is the mean value from mibolerone-treated cells at 120 hours (except for miR-494 which is the mean value from DHT-treated cells at 24 hours). qRT-PCR data normalised to RNU6B. Presented as mean of 3 independent cultures.

Fig.3 Effect of MIB on miR-221 expression (A) Effect of MIB on cell numbers and PSA secretion of LNCaP cells ; Presented as mean of at least six independent cultures with S.D. error bars. Statistical analysis performed by 1-way ANOVA with Dunnett's post hoc test, comparing against the vehicle control where $* * *$ indicates $\mathrm{P}<0.001$, $* * \mathrm{P}<0.01$ and $* \mathrm{P}<0.05$. (B) Measurement of mature miR-221 levels in same samples by qRT-PCR. Data are mean of 3 independent cultures with S.D error bars. Cells treated for 120 hours.

Fig.4 MIB specific induction of the hypoxia-associated miR-210 (120 hours treatment). (A) Comparison of mature miR-210 levels in LNCaP cells in normoxia and hypoxia ( 3 independent cultures); (B) Comparison of VEGF mRNA levels in LNCaP cells in normoxia and hypoxia (3 independent cultures); (C) Measurement of mature miR-210 levels by qRT-PCR in LNCaP cells treated with range of MIB doses; (3 independent cultures (except VC, N=2) (D) Measurement of VEGF mRNA levels by qRT-PCR in LNCaP cells treated with range of MIB doses ( 3 independent cultures 
(except VC, N=2); (E) Pearson correlation between mean miR-210 and VEGF levels in same LNCaP samples treated with range of MIB concentrations. All graphs, errors bars indicate S.D 


\section{Tables}

Table 1 MIB treatment induced changes in miRNA expression

\begin{tabular}{|c|c|c|}
\hline miRNA & Fold change & FDR pval \\
\hline miR-221 & 2.5 DOWN & 0.01592 \\
\hline miR-196b & 1.5 DOWN & 0.15671 \\
\hline miR-1246 & 1.9 DOWN & 0.25493 \\
\hline miR-1290 & 1.8 DOWN & 0.25493 \\
\hline miR-210 & 1.7 UP & 0.25493 \\
\hline miR-182 & 1.3 DOWN & 0.25493 \\
\hline miR-30a & 1.8 DOWN & 0.28308 \\
\hline miR-99a* & 1.2 DOWN & 0.28308 \\
\hline miR-720 & 1.6 UP & 0.28308 \\
\hline miR-105 & 1.1 DOWN & 0.28308 \\
\hline miR-99a & 1.5 DOWN & 0.28308 \\
\hline miR-7 & 1.8 UP & 0.28308
\end{tabular}

Tabulated miRNA are those found to have a significant temporal change in expression levels following treatment with MIB with a false discovery rate (FDR) less than 0.3 between the examined timepoints $(8,24$, and 120 hours of treatment). Values shown are mean fold changes compared to time-matched vehicle control (3 independent cultures per condition ). 
Table 2 DHT treatment induced changes in miRNA expression

\begin{tabular}{|c|c|c|}
\hline miRNA & Fold change & FDR pval \\
\hline let-7e & $2.1 \mathrm{DOWN}$ & 0.27568 \\
\hline miR-16 & 2.4 DOWN & 0.27568 \\
\hline let-7c & $1.5 \mathrm{DOWN}$ & 0.27568 \\
\hline let-7b & $1.3 \mathrm{DOWN}$ & 0.27568 \\
\hline miR-494 & 2.5 UP & 0.27568 \\
\hline miR-200b & 2.3 UP & 0.27568 \\
\hline miR-98 & 2.0 UP & 0.27568 \\
\hline miR-1290 & 1.7 UP & 0.27568 \\
\hline miR-183 & $2.0 \mathrm{DOWN}$ & 0.27568 \\
\hline miR-151-5p & $1.9 \mathrm{DOWN}$ & 0.27568 \\
\hline miR-23a & $2.0 \mathrm{DOWN}$ & 0.27568 \\
\hline miR-107 & $2.2 \mathrm{DOWN}$ & 0.27568 \\
\hline miR-23b & $2.0 \mathrm{DOWN}$ & 0.27568 \\
\hline miR-26b & $2.3 \mathrm{DOWN}$ & 0.27568 \\
\hline miR-195 & $2.0 \mathrm{DOWN}$ & 0.27568 \\
\hline miR-30c & $1.5 \mathrm{DOWN}$ & 0.27568 \\
\hline miR-1260 & 1.7 DOWN & 0.27568 \\
\hline miR-100 & 1.5 DOWN & 0.27568 \\
\hline let-7a & 1.7 DOWN & 0.27568 \\
\hline let-7d & $1.5 \mathrm{DOWN}$ & 0.27568 \\
\hline miR-1280 & $2.3 \mathrm{UP}$ & 0.27568 \\
\hline miR-99a & $1.5 \mathrm{DOWN}$ & 0.27568 \\
\hline miR-30b & $2.1 \mathrm{DOWN}$ & 0.27568 \\
\hline miR-1246 & 1.6 DOWN & 0.27568 \\
\hline miR-125b-2* & $1.5 \mathrm{DOWN}$ & 0.27568 \\
\hline miR-15b & $2.1 \mathrm{DOWN}$ & 0.27568 \\
\hline let-7i & $1.6 \mathrm{DOWN}$ & 0.27568 \\
\hline miR-425 & $1.7 \mathrm{DOWN}$ & 0.27568 \\
\hline miR-24 & 1.9 DOWN & 0.27568 \\
\hline miR-203 & 1.6 DOWN & 0.27568 \\
\hline miR-638 & 1.4 UP & 0.27568 \\
\hline miR-17 & $2.0 \mathrm{DOWN}$ & 0.27568 \\
\hline miR-25 & 1.5 DOWN & 0.27568 \\
\hline miR-148a & $2.1 \mathrm{DOWN}$ & 0.28423 \\
\hline miR-374b & 1.4 DOWN & 0.28423 \\
\hline miR-103 & $2.1 \mathrm{DOWN}$ & 0.28423 \\
\hline miR-15a & $2.0 \mathrm{DOWN}$ & 0.28423 \\
\hline miR-93 & 1.7 DOWN & 0.28423 \\
\hline miR-221 & $1.5 \mathrm{DOWN}$ & 0.28423 \\
\hline miR-200c & 1.8 DOWN & 0.28423 \\
\hline
\end{tabular}

Tabulated miRNA are those found to have a significant temporal change in expression levels following treatment with MIB with a false discovery rate (FDR) less than 0.3 between the examined timepoints $(8,24$, and 120 hours of treatment). Values shown are mean fold changes compared to time-matched vehicle control. (3 independent cultures per condition ). 


\section{Supplementary Figure Legends}

\section{Suppl.Fig.1: Effects of treatments with DHT, MIB, and BIC on LNCaP proliferation.}

In panels (A)-(C) the effects of the three tested compounds on $\mathrm{LNCaP}$ proliferation as measured by alamarBlue ${ }^{\circledR}$ is shown. In panel (D) the ability of a range of BIC doses to inhibit proliferation induced by $2 \mathrm{nM}$ DHT is presented. Shaded area represents 95\% confidence interval for determination of Log EC50 (dotted line). Values are mean \pm S.E.M error bars from 4 independent cultures; Vc is vehicle control; -ve is negative control (media); +ve is $2 \mathrm{nM}$ DHT alone.

\section{Suppl.Fig.2: Effects of treatments with DHT, MIB, and BIC on PSA secretion by} LNCaP.

In panels $(\mathrm{A})-(\mathrm{C})$ the effects of the three tested compounds on PSA secretion are shown (D) the ability of a range of BIC doses to inhibit PSA secretion induced by 2nM DHT is presented. Values are single determinations,

\section{Suppl.Fig.3: EGF secretion in MIB treated LNCaP}

(A) Standard curve of EGF. Data are mean of two replicates per standard; (B) EGF protein expression from MIB-treated LNCaP. Data are mean of seven replicates for samples with S.D. error bars and two replicates for EGF blank (0 pg/mL EGF). 


\section{Figure}

guldA:Revised_Figures.ppt

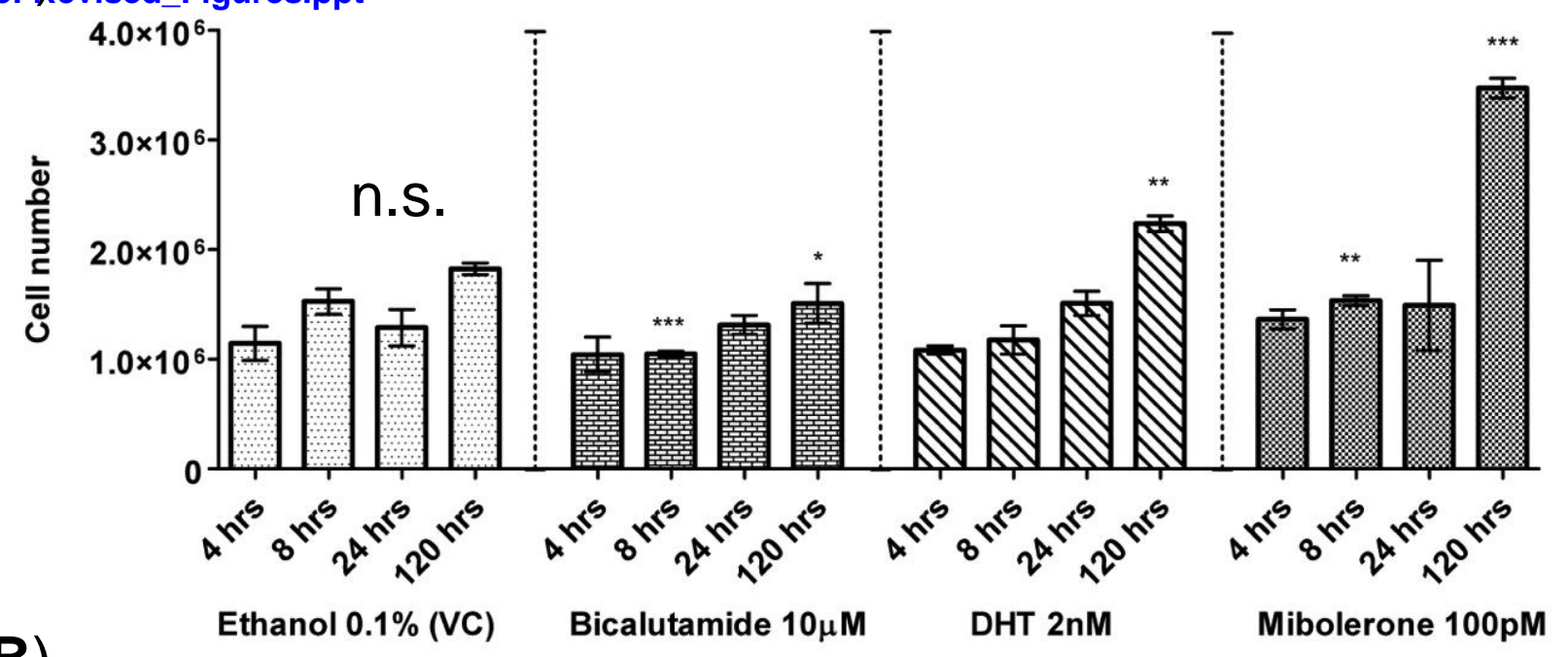

(B)

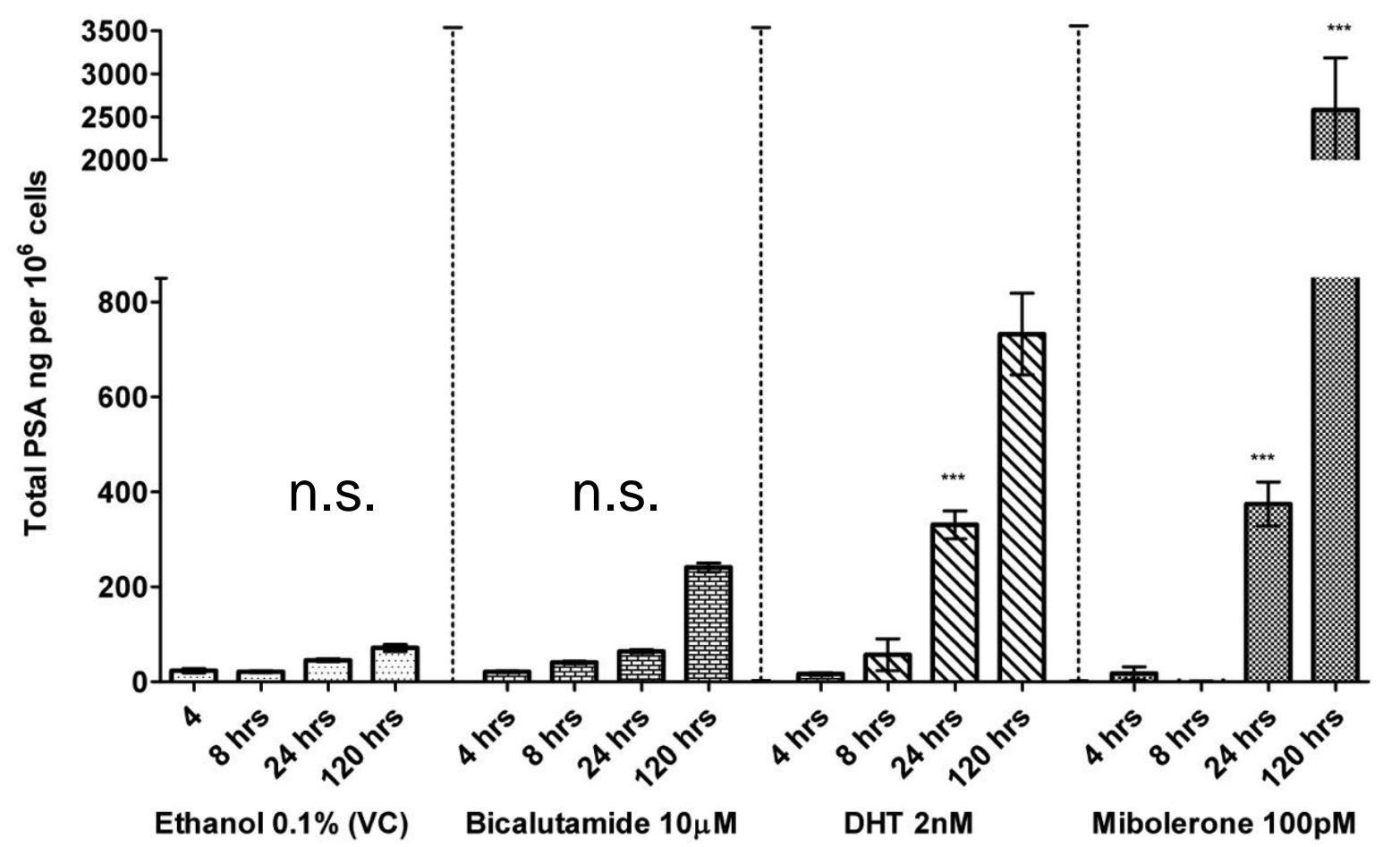

Fig.1 


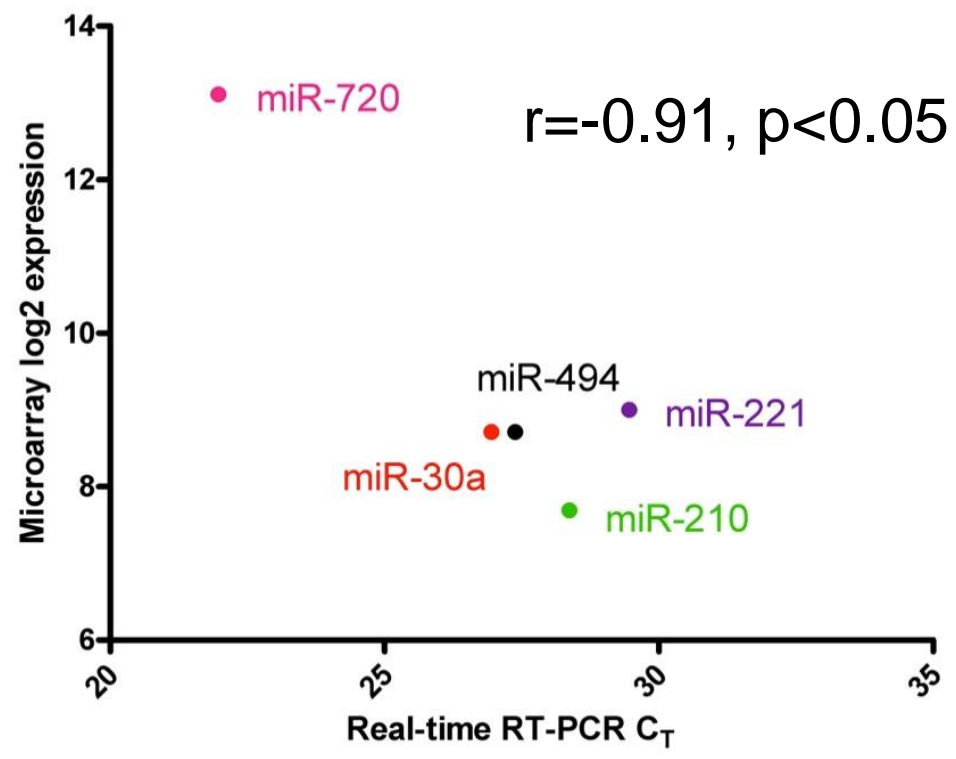

Fig.2 
(A)

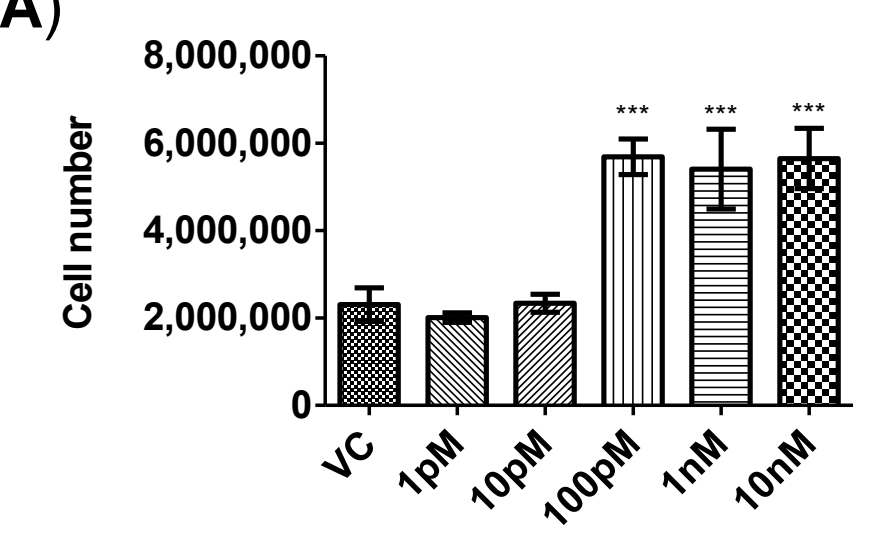

(B)

[Mibolerone]

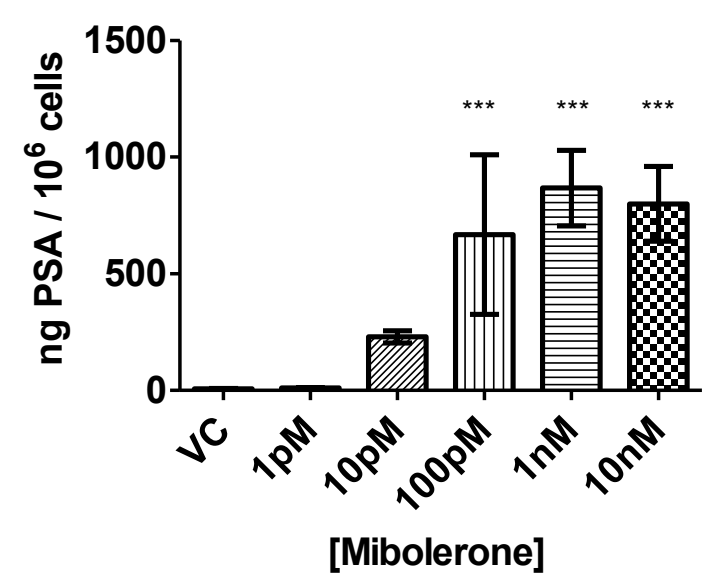

\section{[Mibolerone]}



Fig.3 
(A) miR-210 expression

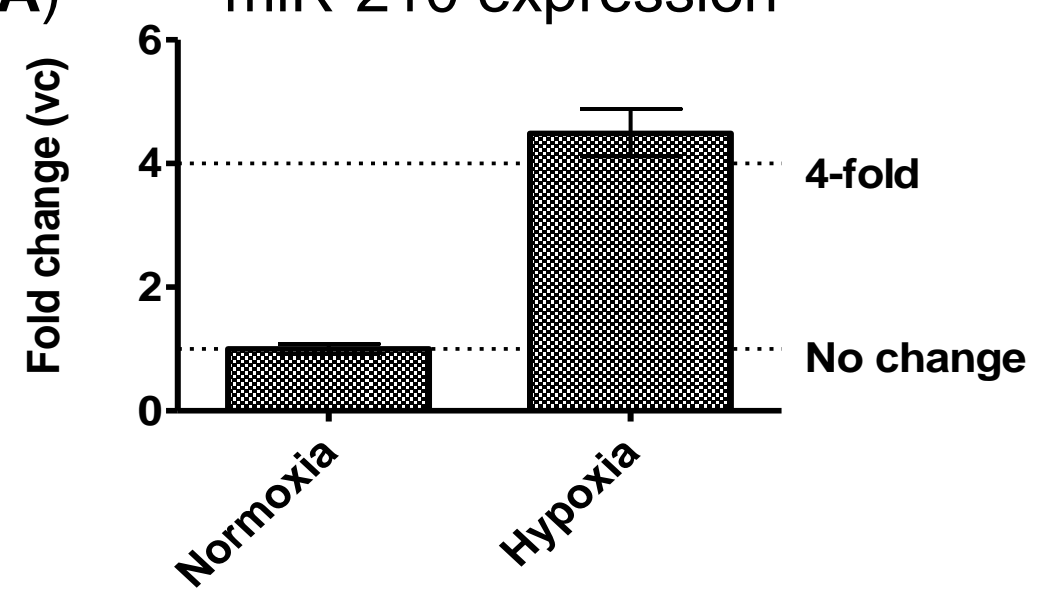

(C)

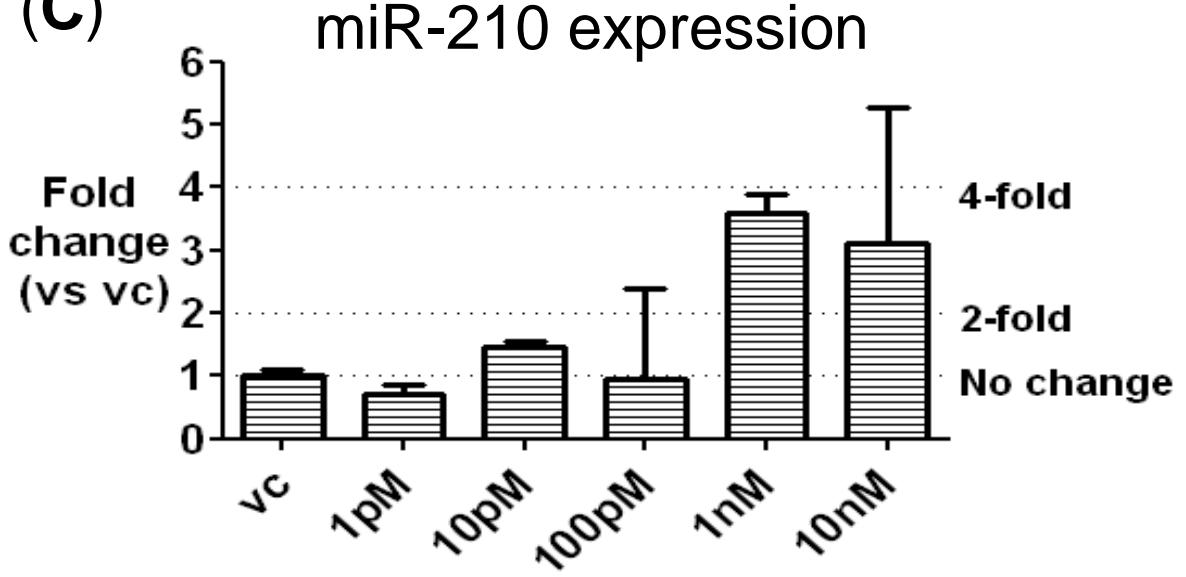

(E)

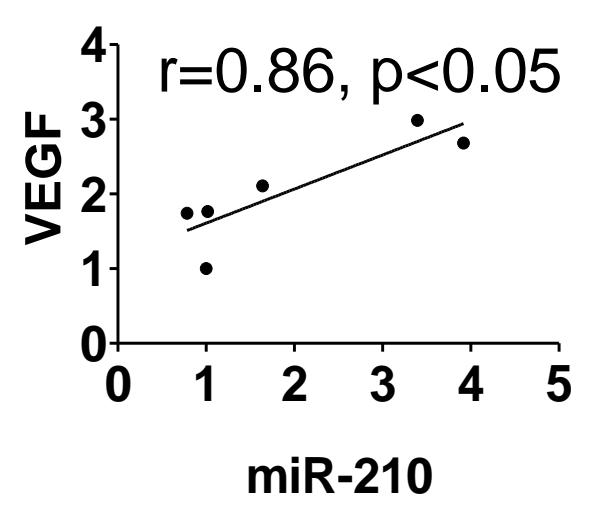

(B) VEGF mRNA levels

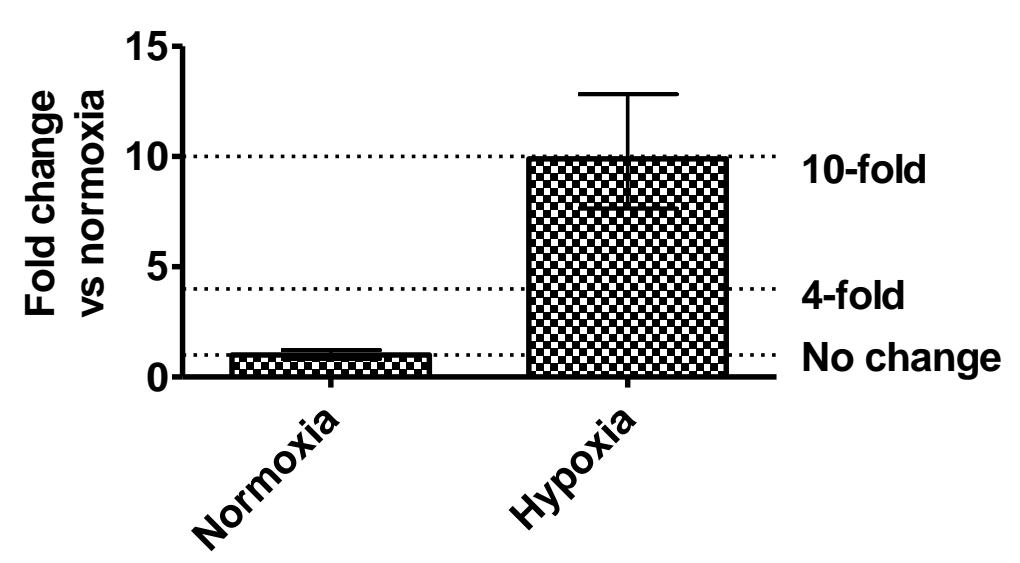

(D) Effect of MIB on VEGF

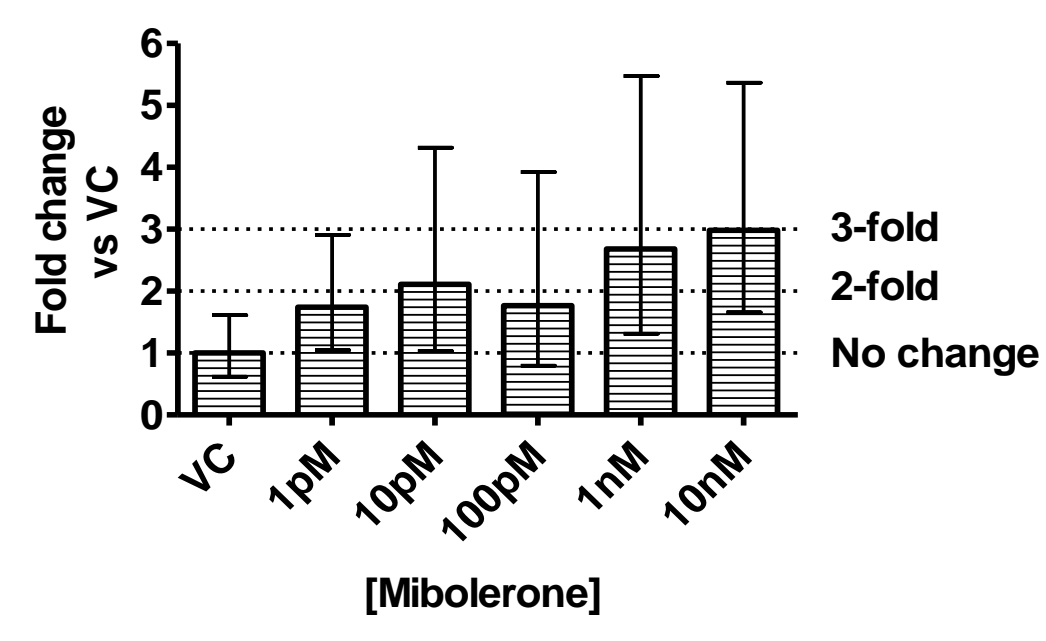

